\title{
Designing Technology for Visualisation of Interactions on Mobile Devices
}

\author{
Kristine Deray \\ University of Technology Sydney (UTS), Australia \\ Kristine.Deray@uts.edu.au \\ Simeon Simoff \\ University of Western Sydney, Australia \\ s.simoff@uws.edu.au
}

Received 1 August 2009; Revised 15 September 2009; Accepted 20 September 2009

\begin{abstract}
Interactions are intrinsic part of what we do. We interact when we work, when we learn, when we visit a doctor, and when we play. With the advent of information and communications technology we can collect rich data (video, audio, and various transcripts including text chat) about such interactions. This opens an opportunity to monitor the dynamics of interactions and to get deeper insights of how they unfold and deliver this information to the interacting parties. This paper presents the design of a technology for visualising information about the dynamics of unfolding of interactions and presenting it in an ambient display on mobile devices. The purpose of this technology is the delivery of such information to the point of decision making.

Categories and Subject Descriptors: Information and Communications Technology [Mobile Visualisation]: Visual Analytics

General Terms: Information Visualisation, Visual Reasoning, Visual Expression

Additional Key Words and Phrases: Monitoring Interactions, Health Informatics
\end{abstract}

\section{INTRODUCTION}

Many human endeavours are based on some form of collaboration and cooperation. In healthcare, practitioners and patients collaborate to achieve a way of improving the patient's state of health. In a participatory design, designer(s) and client collaborate to develop the design that best suits the client. In science and technology, researchers from different disciplines collaborate in order to find innovative solutions.

Collaboration relies on interactions; hence, there is a benefit if there exists a way

Copyright(c) 2009 by The Korean Institute of Information Scientists and Engineers (KIISE). Permission to make digital or hard copies of part or all of this work for personal or classroom use is granted without fee provided that copies are not made or distributed for profit or commercial advantage and that copies bear this notice and the full citation on the first page. Permission to post author-prepared versions of the work on author's personal web pages or on the noncommercial servers of their employer is granted without fee provided that the KIISE citation and notice of the copyright are included. Copyrights for components of this work owned by authors other than KIISE must be honored. Abstracting with credit is permitted. To copy otherwise, to republish, to post on servers, or to redistribute to lists, requires an explicit prior permission and/or a fee. Request permission to republish from: JCSE Editorial Office, KISE. FAX +8225211352 or email office@kiise.org. The Office must receive a signed hard copy of the Copyright form. 
of monitoring, understanding and evaluating interactions that occur during collaboration and feeding that evaluation back into the interaction process, in order to improve the process of collaboration and its outcomes. The structure and content of interactions can reveal different facets of collaboration. For example, take a collaborative design in an educational setting, where the structure of interactions between team members provides information about the style of collaboration. A team can be well interlinked and develop a deep shared understanding of the problem or the collaboration can be reduced to a loose coordination of individual work without necessarily developing a shared understanding of the problem [Simoff and Maher 2000]. Parameters like the number, length and content of messages in an interaction have been used to identify emergent leaders in asynchronous and synchronous virtual environments [Simoff and Sudweeks 2007]. These studies are examples of what typically has been studied in interactions, namely (i) communication behaviours of participants (for example [Bochner et al. 1977]); (ii) styles of communication and interaction (spanning from studies in conversational interactions [Tannen 2005] and their support [Bradner et al. 1999] to gesture-based interactions [Dicke et al. 2008] and support for social cues [Louwerse et al. 2005]; and (iii) various networks, derived from social interactions between individuals [Golder et al. 2007]. The studies so far have not focused on extracting information about the process, the dynamics of interactions and how to utilise that information in order to improve the process itself.

\section{1 inferactions as a Process and the Delivery of Information About Such Pro- cess}

Interactions constitute a process that unfolds. The way interactions unfold can tell us a lot about the interactions themselves. Hence, in order to improve processes that rely on interactions, it is important to develop methods of encoding the way interactions unfold, capable of representing that information and allowing both humans and machines to utilise such information during the interaction process in order to improve interactions and respective processes. For example, in health care, the treatment process is expected to benefit if we can present the information about how patientpractitioner interactions unfold, and if, based on that representation, one can judge in relation to whether there has been a good communication or not, and if not, where were the bottlenecks [Deray and Simoff 2007]. In design, the participatory design process may benefit if we can present the information about how client-designer interactions unfold and if, based on that representation, one can judge about whether design communication has been good or not, and use the interaction patterns to align with the emergence of design solutions, in order to revisit intermediate design solutions [Simoff and Maher 2000]. In mobile education, for instance, work and lifebased learning environments, there is a variety of interactions [Billet 2008]. These include (i) interactions between the learner and people involved in different roles in the learning processes; (ii) interactions with various learning materials and the learning environment; and (iii) interactions that are part of the learning content. For example, in health-care and nursing [Gabb and Keating 2005], the work-based mobile learning process and practice involve interactions between nurses and health-care practitioners on the one side and their patients on the other side. 
In these examples the timely delivery of the information about interactions, the interaction profile, to the decision-making point is vital. The actual process and means of delivery are ambient to the decision-making, which implies that the displays delivering information about interactions are expected to have a certain degree of:

- ambience - in not obstructing the actual interaction process;

- pervasiveness - in being embedded not only within the environment [Greenfield 2006] but within human cognitive capabilities; and

- compactness - in being able to present the information in a way that can be grasped 'from a glance.'

Technologically the problem implies the development of an ambient information system about interactions that communicates information to the involved parties in a visual manner. In the design process we take into account the taxonomy of ambient information systems presented in [Pousman and Stasko 2006].

\subsection{Information Design of Visual Displays for Mobile Devices}

Mobile devices are rapidly expanding both their diversity in terms of capabilities and sizes, and their 'operational' territory, including the facilitation of decision-making, by their ability to deliver information to the point of decision-making. The diversity of the application fields spans from management in diverse business areas through educational areas and to the delivery of health care. The changes in the business processes, caused by these technological developments, reflect back on the technology development, increasing the requirements in terms of capabilities. For designers of mobile devices essential requirements are:

- communication design - the hardware and software design enabling smart network communication capabilities, and;

- information design of the application displays, enabling efficient interaction and analytics capabilities of respective mobile devices.

These requirements face the constraints on the size of the mobile devices, and, in particular, on their displays and interface controls.

Decision-making as a process involves variety of information, demanded at different levels of granularity. Visual representations translate data and information into a visible form that highlights important features, including commonalities and anomalies. Such visual representations make it easier for analysts and decision makers to grasp key aspects of presented information quickly [Thomas and Cook 2005].

The design of visual representations of information has been the focus of the fields of scientific data visualisation, information visualisation and design computing. All three fields create graphical models and visual representations from data that support direct user interaction. In the first two fields the aim is to explore large data sets and information spaces acquiring insights into useful information embedded there [Ferreira de Oliveira and Levkowitz 2003]. In scientific data visualisation graphical models are derived from measured or simulated data, which represent concepts associated with objects in the physical world. Yet in information visualisation, the graphical models may represent abstract concepts and relationships that do not necessarily have a 
counterpart in the physical world. In many visualisations, both approaches use concepts from a commonly known theme to express the semantics of domain data in popular terms. For instance, landscape is a popular theme for visualising diverse data sets, ranging from climate data sets to large collections of text documents (for example, high peaks in the IN-SPIRE ${ }^{T M}$ document landscape visualisation show prominent topics, and peaks that are close together represent clusters of similar documents [Thomas and Cook 2005]). The third field, design computing, deals with the visualisation of physical objects, for example, building and interior design models, and abstract concepts, for instances, spaces in virtual worlds. These visualisations aim to represent different aspects of designs, facilitating the development and evolution of design ideas [Maher et al. 2000].

All these areas are related to the work presented in this paper as they have led to some schemes to visualise communication and interaction data. The tendency so far has been to present interactions in terms of the results they produce rather than to provide the mechanisms to encode and display 'how' interactions unfold over time [Deray and Simoff 2007]. For example, a network type of visualisations focuses on displaying the relations between interacting entities. In [Simoff and Maher 2000] authors present a method for depicting patterns in unfolding of collaboration within teams, based on a nested-rectangles visual representation of on-line communication utterances between team members.

Creating well-constructed and consistent visual representations remains a challenge (see Chapter 3 in [Thomas and Cook 2005] for some of the issues facing designers of visual representations). So far the creation of visualisations has been essentially an ad-hoc process. There has been a little attention to the in-depth application of formal design methods to the design process, engineering as a technology and consistent evaluation [Ferreira de Oliveira and Levkowitz 2003]. In [Deray and Simoff 2007] the authors presented a framework for designing visual languages based on the physicality of human movement and demonstrated its application with an example of constructing a visual language. The language utilised the strength of humans in recognizing intuitively constructs in interactions modeled on human movement. In this paper we consider the extension of the design method with capabilities that cater for the design and development of visualisations and respective visual languages in the context of mobile computing and the delivery of the visual expressions on mobile devices.

The physicality of human movements provides us with the basis for modelling the domain of interactions. The visual elements, including their form and the rules of their behaviour, are developed through understandings derived from the intrinsic dimensions and qualities of the system of human movements. The argument for utilising human movement constructs places the body as central to understanding and deriving the connecting metaphor for the domain of interactions [Pheifer and Bongard 2007].

This paper continues and extends the work presented by the authors in Deray and Simoff 2008]. Of note are the following:

- This work considers how information about the dynamics of interactions can be presented as ambient display on mobile devices at the point of decision-making. 
For the purpose, the paper takes into account ambient considerations of display in the design stage in order to comply with the requirements of pervasiveness and compactness.

- Subsequently the above leads to the extension of requirements of the design principles of visual representations for mobile devices, established in [Deray and Simoff 2008]. Three new design principles are now included, namely, complexity, expandability and scalability, in the context of displays operating in mobile devices.

- The paper considers another group of additional design principles, which address the design of ambient aspects of delivery. These are, information capacity, attention attracting capacity, expressive power and aesthetics.

- As mobile devices provide access to various social networking technologies and even 3D virtual worlds, the paper extends the the formalism that drives the computational models of the qualities of both 'Rising and Sinking' and 'Contraction and Extension' elasticities. Originally, the expressions considered only time as the argument for the measures of length of actions. However, in text-based communication, depending on the definition of action, the length of an action may be measured in terms of the number of words in the message. In this paper, the models of the qualities are presented in the general terms of length, opening the approach beyond the analysis of face-to-face interactions.

- The conceptual modelling of the visual elements is explained in more detail in this paper, tracing how the elements of human movements influence the visual representations of the qualities of the 'Rising and Sinking' and 'Contraction and Extension' elasticities.

- The demonstration of the analysis and application of this technology to case studies in health is expanded with the presentation of a broader range of views.

\subsection{Organisation of the Paper}

Further the paper is organised as follows. In Section 2 we present the design principles of visual representations of interactions for mobile devices. In Section 3 we present a methodology for designing representations of information about interaction, including an example of the underlying computational and visual models of such representation. Section 4 presents the deployment of these visual models on mobile devices, using cases of their application in the healthcare domain in order to demonstrate the range of functionalities that support visual analysis of the dynamics of practitioner-patient interactions. Section 5 concludes the paper with a summary of the developments and discussions of the limitations of the approach.

\section{DESIGN PRINCIPLES OF VISUAL REPRESENTATIONS OF INTERAC- TIONS FOR MOBILE DEVICES}

In order to visualise information about real-time dynamics of interactions on a static mobile display in an ambient manner, we formulate the design principles that are the guiding posts in the practical development of the overall method, respective visualisation and visual language. In [Deray and Simoff 2007] the authors have specified a set of principles for the design of the visual components (primitives and 
their aggregations) for constructing a visual language based on the elements of human movements. These principles, an adaptation of the requirements enlisted in Pretorius 2005], include the following: symmetry, regularity, similarity, connectivity, legend, containment and aggregation, and transparency. These criteria are addressed in details in [Deray and Simoff 2007].

In this paper, we extend this list with two groups of additional design principles. The first group is related to making a visualisation suitable for mobile devices. This additional properties, which bear some similarity to the requirements towards the "parallel coordinates" visualisation [Inselberg 1997], include the following principles.

Complexity: This design principle aims at low representational complexity, so that through the visual representation the development and unfolding of interactions can be monitored in real-time on a mobile device.

Expandability: This design principle aims at visualisation that can accommodate changes in the representation of interaction information.

Scalability: This design principle addresses not only the size of the data displayed, but the diversity of screens on which visualisation can appear, ensuring the displayed components remain recognisable under projective transformations, such as scaling, rotation, translation or if presented in perspective.

The second group of design principles, adapted from [Pousman and Stasko 2006], adds the ambient aspects of delivery of the information about interactions through the visualisation. These include the following principles:

Information capacity: This design principle is related to the trade-off between the size of the visual element, the space for the display of the elements and the time for presenting an information segment.

Attention attracting capacity: This design principle is related to the ability of visualisation and a respective visual language to demonstrate critical patterns in interaction, capable of rising person's alert and the need for an immediate consideration during the decision making.

Expressive power: This design principle relates to the semiotics [Chandler 2004] of the discrete elements that constitute the visualisation and their combinations that constitute the visual language, i.e., how the data about interactions are encoded into patterns, pictures, words, or sounds that eventually convey the information about interactions. Such visual semiotics is directly related to how condensed the information is delivered by the visualisation. The range is from direct presentations of low level data for monitoring purpose to metaphors (e.g. Cherno. faces [Cherno 1973]) and other graphical displays of complex and latent information structures (see [Chen 2004] for a survey of diverse displays) that convey condensed information.

Aesthetics: This design principle is concerned with to what extent a graphical display is being visually pleasing. Extraction and sense making of information about interactions are related to the ability to gain insights; hence, this design principle is closely connected with the principles of information capacity and attracting attention capacity.

The quest for implementing these principles acts as a constraint factor in the development of the methodology for designing computational representations and visualisations of information about interactions, presented in the next section. 


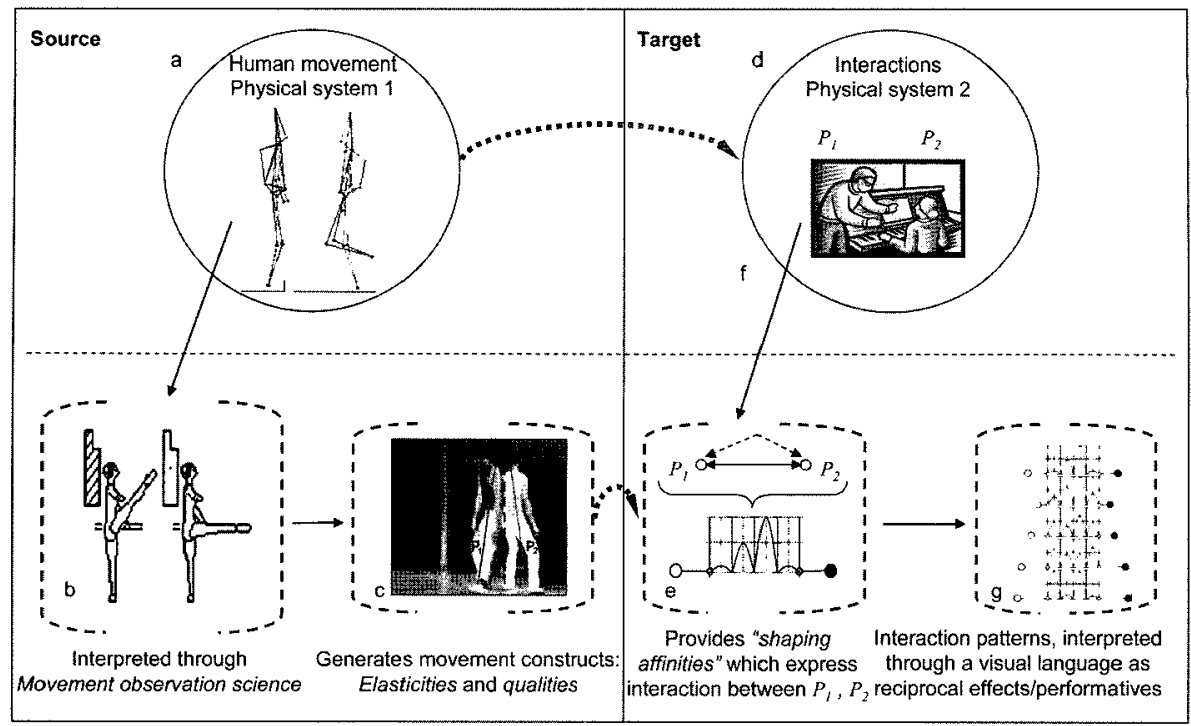

Figure 1. Mapping the Domains of Human Movements and Interactions as Two Physical Systems.

\section{METHOD FOR DESIGNING COMPUTATIONAL REPRESENTATIONS AND VISUALISATIONS OF INFORMATION ABOU'T INTERACTIONS}

The proposed visualisation methodology relies on consistent semantic mapping and rigorous underlying formalisms. Figure 1 presents compactly the conceptual modelling that enables the method for designing representations of interactions following the elements of the physicality of human movements. The modelling approach views both domains as physical systems. Then the systematic mapping between the domains, inspired by Lakoff and Johnson's approach to metaphors [Lakoff and Johnson 1980], expresses the target domain (d) - the physical system of interactions, through the constructs of the source domain (a) - the physical system of human movements (indicated by the arrow in the upper half of Figure 1). Figure 1 also summarises the process. The source domain - "human movement" (a), is interpreted and formalised through the methodology developed in Movement observation science (b) [Newlove and Dalby 2004]. The derivation of the constructs of elasticities and qualities (c), which are central to the development of computational representations of interactions and respective visualisations, is achieved by the application of elements of Movement observation science to the analysis of contact improvisation data. The two groups of constructs provide the forms and behaviour rules of the shaping affinities for expression of information about interactions (e). The target domain (d), the domain of interactions between two or more parties, is expressed then in terms of the shaping affinities (f), whose behaviour follows the computational models of the elasticities and qualities. These models are computed as functions of interaction parameters. The visual language utilises these values to express the patterns and reciprocal effects of interaction (g) between involved parties [P1 and P2 in Figure 1] in terms of patterns of behaviour of the constructs.

The mapping, illustrated by the process in Figure 1, takes concepts that describe 
Table I. An Overview of the Key Groups of Constructs Derived from the Source Domain of Human Movement and Relating Them to Their Function in the Target Domain of Interaction Representation.

\begin{tabular}{|l|l|l|}
\hline \multicolumn{1}{|c|}{$\begin{array}{c}\text { Source domain } \\
\text { constructs }\end{array}$} & \multicolumn{1}{|c|}{$\begin{array}{c}\text { Target domain } \\
\text { constructs }\end{array}$} & $\begin{array}{l}\text { Using constructs from the source domain to } \\
\text { describe constructs in the target domain }\end{array}$ \\
\hline (a) Elasticities & (c) Reciprocal effects & $\begin{array}{l}\text { Elasticities represent the reciprocal effects as } \\
\text { integral dimensions of the domain of interaction. }\end{array}$ \\
\hline (b) Qualities & (d) Performatives & $\begin{array}{l}\text { Qualities represent cooperative processes, } \\
\text { cooperation interpreted as "common ground" } \\
\text { between parties in the interaction domain }\end{array}$ \\
\hline
\end{tabular}

two frames of reference of human movement; (i) body position [the place of the body in space]; and (ii) body dynamics [the motion that causes and expresses the change from one position of the body to another], and uses their computational representations in the interactions domain. The interaction parameters that are used to compute these representations depend on the nature of the interactions. For example, modalities in face-to-face interactions differ from the modalities of interactions in virtual worlds. In face-to-face interactions parameters used to compute the models of elasticities and qualities can be various functions of time and frequency of individual actions in the interactions. In interactions in virtual worlds parameters used to compute the models of elasticities and qualities can include various statistics of chat logs (for example, utterance length, frequency and density), parameters of avatar's gestures and other modes of behaviour.

Table I illustrates the linking of the key groups of constructs in the source and target domains in Figure 1, and briefly describes the way these concepts are related in the context of their function in the target domain. The behaviour of elasticities describes the reciprocal effects between parties in human movement interaction. How the interaction functions, for instance, is independent from other interactions, it is symmetrical or asymmetrical, balanced or unbalanced - all these are expressed by the changing shape and structure of the elasticities. Qualities generate a canonical structure in both domains. Similar to the use of the term in music, where a number of instruments or voices contribute to the overall structure with some modification in a dimension such as pitch, the basic idea is that parts combine to make the whole and that each part expresses a variable on a dimension that is integral to the domain. In the target domain this notion references the concept of a "common ground" defined as an aggregated measure derived from the dynamics of participating parties in interactions.

The set of constructs considered in this paper include the following elasticities the rising and sinking $(R-S)$ elasticity and the contraction and extension $(C-E)$ elasticity. As noted we derive the rules of behaviour of the visual elements from the respective physical movement of parties involved in contact improvisation.

As form and function are intrinsically coupled in human movement, on the one hand, and, the purpose of the visualisation system is to allow monitoring interactions and depict their patterns in terms of the meta-information extracted, we have adapted the "Form-Semantics-Function" methodology for development of visualisation techniques 
Table II. Deriving the Forms of the Basic Elasticities from the Source Domain Based on the Corresponding Anatomical Actions.

\begin{tabular}{|l|l|l|}
\hline Elasticity & Contraction and Extension & Rising and Sinking \\
\hline Form & & \\
\hline Semantics & $\begin{array}{l}\text { The amplitude on the horizontal axis } \\
\text { indicates the strength of interaction. In } \\
\text { terms of interpretation, it models } \\
\text { strength as states of attraction, repulsion } \\
\text { or stable behaviour. }\end{array}$ & $\begin{array}{l}\text { The amplitude on the vertical axis } \\
\text { indicates the elasticities that stretch } \\
\text { up and sink down. }\end{array}$ \\
\hline Function & $\begin{array}{l}\text { Describes the intensity of the interaction. } \\
\text { Describes flow and effort of the } \\
\text { interaction. }\end{array}$ \\
\hline
\end{tabular}

for visual data mining and analytics [Simoff 2001, 2008]. Table II presents an example of the formal derivation of the form and behaviour constraints for the $R-S$ and $C-E$ elasticities from the movement analysis of contact-improvisation data. As indicated by the double sided arrows, the action of the two elasticities has been separated in vertical and horizontal directions, respectively, spanning a $2 \mathrm{D}$ representation of interactions.

Elasticities are descried through the computational models of their qualities. In its current development the $R$-S elasticity includes four qualities, presented here in terms of their interpretation in the interaction domain. Flow $\left(q_{1}^{R-S}\right)$, which characterises the obstruction (e.g., language/social/cultural, etc); transition $\left(q_{2}^{R-S}\right)$, which characterises interaction in time; exertion $\left(q_{3}^{R-S}\right)$, which correlates to the amount of effort required for an interaction to achieve some perceived position; and control $\left(q_{4}^{R-S}\right)$, which indicates the amount of control applied in the interaction. The $C-E$ elasticity currently includes a single quality - intensity $\left(q_{1}^{C-E}\right)$, which indicates the strength of the interaction.

\subsection{Computational Models of the Qualities of $R-S$ and $C-E$ Elasticities}

Interactions are treated as a sequence of actions, where each action belongs to one of participants in the interaction. In [Deray and Simoff 2007] we have presented the computational models of all qualities of $R \cdot S$ and $C-E$ elasticities, except the model of the flow quality in the $R$-S elasticity. The later has been left as a categorical value with three possible values. In [Deray and Simoff 2008] we introduced the missing computational model for the flow quality. As the derivation of these initial models has been influenced by the data of face-to-face interactions, in both [Deray and Simoff 2007] and [Deray and Simoff 2008] expressions are functions of time.

In this paper we demonstrate how qualities are computed in order to behave according to the descriptions presented in Table II. We also present a more general 
form of the models, which are suitable for different interactions formats, including online interactions. We follow the order of the introduction of the qualities, starting with the quantitative representation of the flow. For that let's consider an interaction $\mathrm{j}$ between two parties $P_{k}$ and $P_{l}$. Let $n_{k}$ and $n_{l}$ be the number of actions in the interaction $j$ for each participant, respectively.

Flow $\left(q_{1}^{R-S}\right)$ is a measure of the contribution of each participant to an interaction. To measure the flow we consider the difference between the lengths of each participant's actions in time. Flow is proportional to the difference between the relative values of the length of participant's actions. We consider that each participant contributes a number of actions to the interaction. Let's consider the lengths of contributing actions in an interaction $j$ between two parties $P_{k}$ and $P_{l}$. In this case, the length of the action is proportional to the action time - the time it takes for an action to be performed. The compound length of an individual action includes the reaction time (the delay, before taking the action) and the actual action time. Let $t_{i}\left(P_{k}\right)$ and $t_{i}\left(P_{l}\right)$ be the respective action times contributed by $P_{k}$ and $P_{l}$ to the interaction $j$. Then the lengths of respective actions are $l e n_{i}\left(P_{k}\right)=t_{i}\left(P_{k}\right)$ and $l e n_{i}\left(P_{l}\right)=t_{i}\left(P_{l}\right)$.

The flow Flow $_{j}\left(P_{k}, P_{l}\right)$ of the interaction $j$ is defined as

$$
\operatorname{Flow}_{j}\left(P_{k}, P_{l}\right)=\frac{1}{\max \left\{n_{k}, n_{l}\right\}} \sum_{i=1}^{\max \left\{n_{k}, n_{l}\right\}}\left[1-\frac{\mid \operatorname{len_{i}}\left(P_{k}\right)-\operatorname{len}\left(P_{i}\right)}{\mid \operatorname{len} n_{i}\left(P_{k}\right)+l e n_{i}\left(P_{l}\right)} \mid\right]
$$

The measure of flow, defined in equation 1 , reflects the domination of interaction by a party with a large number of actions and greater length of the actions than those contributed by the other party.

Transition $\left(q_{2}^{R-S}\right)$ is proportional to the length of the interaction. Let $\operatorname{Len}_{j}\left(P_{k}\right)=$ $\sum_{i=1}^{n_{k}} \operatorname{len}_{i j}\left(P_{k}\right)$ and $\operatorname{Len}_{j}\left(P_{l}\right)=\sum_{i=1}^{n_{l}} \operatorname{len}_{i j}\left(P_{l}\right)$ be the compound lengths of all individual actions of $P_{k}$ and $P_{l}$ during the interaction $j$. Then the normalised average lengths of

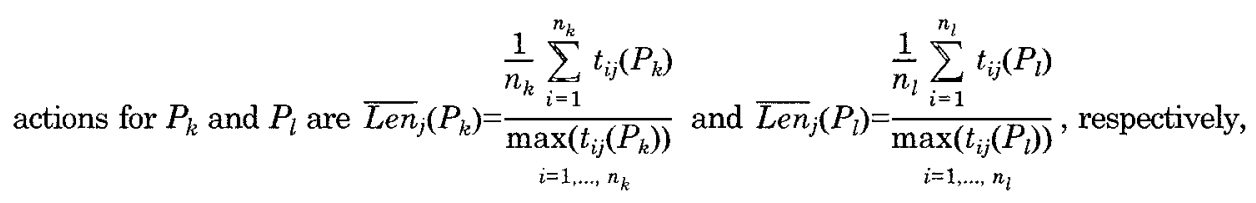
and the average normalised length of an action in the interaction is $\overline{\operatorname{Len}}_{j}\left(P_{k}, P_{l}\right)=$ $\overline{\operatorname{Len}}_{j}\left(P_{k}\right)+\overline{\operatorname{Len}}_{j}\left(P_{l}\right)$. Then the transition of interaction $j$ is computed as

$$
\text { Transition }_{j}\left(P_{k}, P_{l}\right)=1-0.5 * \overline{\operatorname{Len}}_{j}\left(P_{k}, P_{l}\right)
$$

Exertion $\left(q_{3}^{R-S}\right.$ ) is correlated with the amount of effort required for the interaction to proceed to some perceived position. For example, in the case of healthcare interactions, perceived position may refer the patient's progress -that is an identifiable landmark. This could be a test, a drug administration, a consultation or part of a diagnosis, and so on. The measure of exertion for interaction $j$ relates the duration (length proportional to time) of interaction $j, \operatorname{Len}_{j}\left(P_{k}, P_{l}\right)=\operatorname{Len}_{j}\left(P_{k}\right)+\operatorname{Len}_{j}\left(P_{l}\right)$, to the maximum duration in the considered set of interactions $\max _{j}\left\{\operatorname{Len}_{j}\left(P_{k}, P_{l}\right)\right\}$. 
Then the exertion of interaction $j$ is computed as

$$
\text { Exertion }_{j}\left(P_{k}, P_{l}\right)=1-\frac{\operatorname{Len}_{j}\left(P_{k}, P_{l}\right)}{\max _{j}\left\{\operatorname{Len}_{j}\left(P_{k}, P_{l}\right)\right\}}
$$

Control $\left(q_{4}^{R-S}\right)$ is derived from the analysis of the interaction content by evaluating how flexible is the context that the interaction is embedded into. This is related to the number of possible paths or decision forks the players have between them for progress to occur. In its simplest form, for a given interaction this can be derived from comparison between the number of iterations Iterations $s_{j}\left(P_{k}, P_{l}\right)$ in a given interaction $j$ compared to the largest number of iterations $\max _{j}\left\{\right.$ Iterations $\left.s_{j}\left(P_{k}, P_{l}\right)\right\}$ in the considered set of interactions,

$$
\text { Control }_{j}\left(P_{k}, P_{l}\right)=\frac{\text { Iterations }_{j}\left(P_{k}, P_{l}\right)}{\max _{j}\left\{\text { Iterations }_{j}\left(P_{k}, P_{l}\right)\right\}}
$$

Intensity $\left(q_{1}^{C-E}\right)$ indicates the strength of the interaction. By analogy with the intensity of movement, Intensity ${ }_{j}\left(P_{k}, P_{l}\right)$ of interaction $j$ is proportional to the number of actions $n_{k}$ and $n_{l}$ of respective individual parties $P_{k}$ and $P_{l}$ during the interaction,

$$
\text { Intensity }_{j}\left(P_{k}, P_{l}\right)=1-\frac{\left|n_{k}-n_{l}\right|}{\max \left\{n_{k}, n_{l}\right\}}
$$

\subsection{Visual Models of the Qualities of R-S and C-E Elasticities}

Equations 1.5 produce the values that drive the behaviour of the visual elements.

The design of the visual elements of a system for visualising information about the interaction process is guided by:

- the visual behaviour guidelines presented in Table II;

- the design principles formulated in Section 2.

Figure 2 presents diagrammatically the application of our approach, shown in Figure 1, to the design of the visual representation of the qualities of respective

Table III. Composition Rules for the Visual Representations of the Qualities of the Elasticities.

\begin{tabular}{|c|l|l|}
\hline Rule & Visual element of the $C-E$ elasticity & Visual element of the $R-S$ elasticity \\
\hline 1 & $\begin{array}{l}\text { Each arm of } q_{i}^{C-E} \text { starts at the internal } \\
\text { border of the track segment and expands } \\
\text { towards the external border. }\end{array}$ & $\begin{array}{l}\text { Each curve representing the value of } q_{i}^{R-S} \text { is } \\
\text { symmetric with respect to the vertical axis } \\
\text { passing through the middle of the cell. }\end{array}$ \\
\hline 2 & $\begin{array}{l}\text { The arms that represent the first quality } \\
q_{1}^{C-E} \text { are aligned with the lower end of the } \\
\text { element; the anchor points are attached to } \\
\text { the arms of } q_{1}^{C-E} \text {. }\end{array}$ & $\begin{array}{l}\text { The cell that represents the first quality } \\
q_{1}^{R-S} \text { is aligned with the left end of the } \\
\text { effort-shape element. }\end{array}$ \\
\hline 3 & $\begin{array}{l}\text { The arms that represent each subsequent } \\
\text { quality } q_{i}^{C-E} \text { are positioned above the } \\
\text { previous arm pair; the correspondence of } \\
\text { position to the index number does not } \\
\text { change. }\end{array}$ & $\begin{array}{l}\text { Each cell that represents a subsequent } \\
\text { quality } q_{i}^{R-S} \text { of the } R-S \text { elasticity is } \\
\text { positioned to the right of the previous cell; } \\
\text { the correspondence of position to the index } \\
\text { number does not change. }\end{array}$ \\
\hline
\end{tabular}




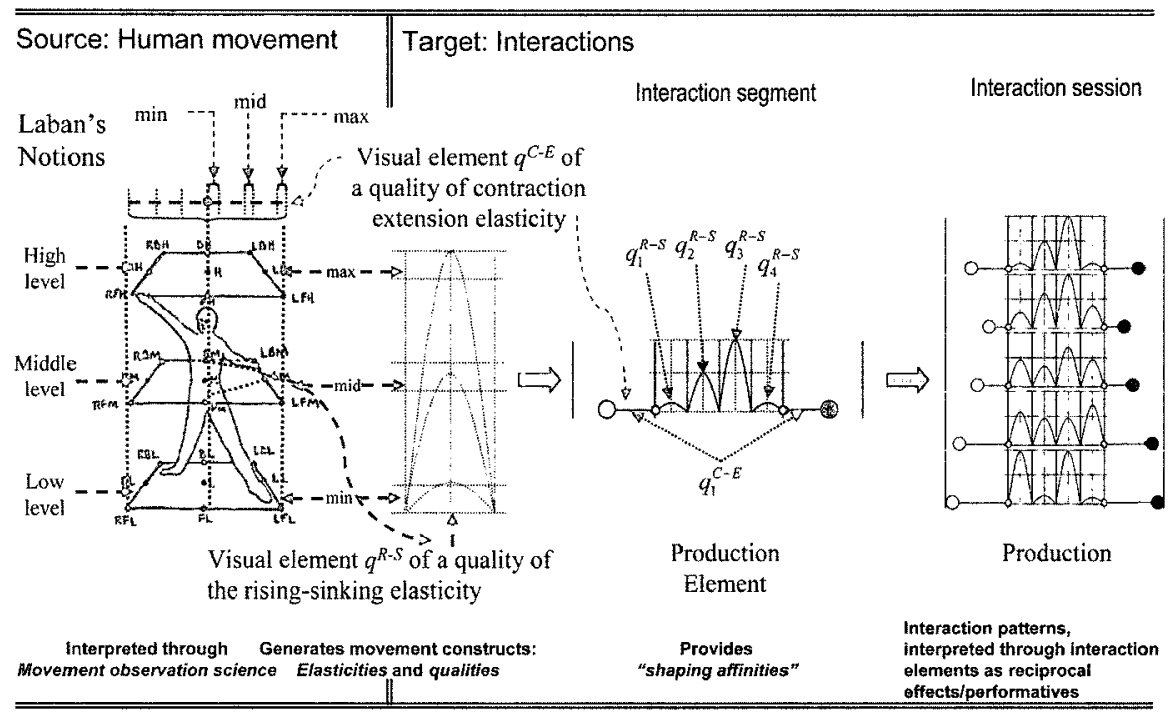

Figure 2. The Elements Encoded in the Physicality of Human Movement and Respective Constructs, Extracted from Movement Observation Science Provide the Basis for Defining the Shape, Behaviour and Aggregation of the Constructs of the Visual Representation of Interactions.

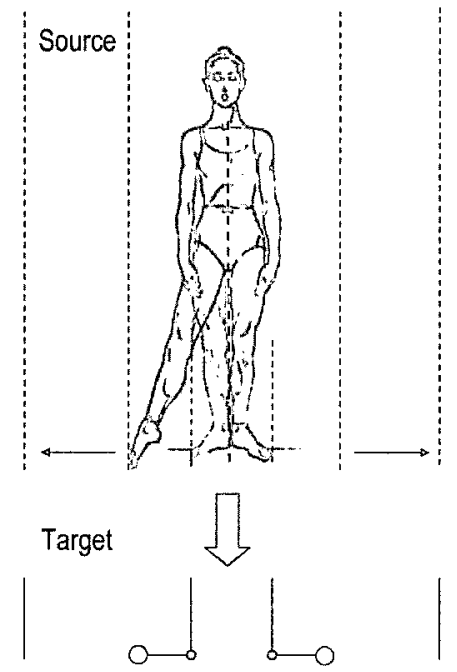

a. Deriving the visual representation of $q_{i}^{C-E}$
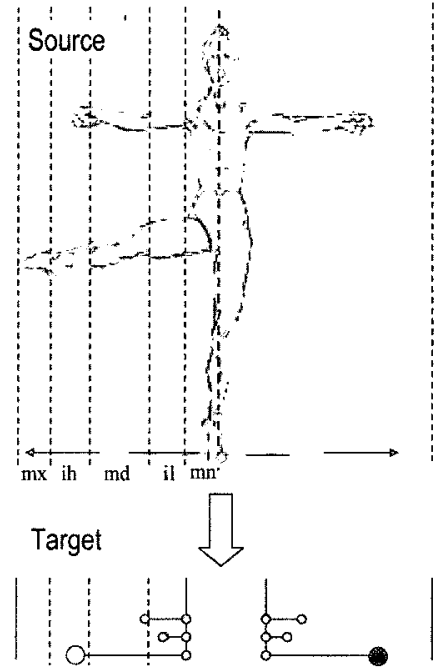

b. Ranges and positioning of qualities $q_{i}^{C-E}$

Figure 3. Derivation of a Visual Element Representing a Quality of the $C-E$ Elasticity, the Ranges of its Values, and the Positioning of Visual Elements Representing Different Qualities $q_{i}^{C-E}$ of the $C-E$ Elasticity.

elasticities, identified in Laban's system. The main rules for constructing the visual elements, derived from Laban's system and addressing the Form-Semantics-Function properties described in Table II, are summarised in Table III.

Figure 3 presents in more details how the elements of human movement influence 


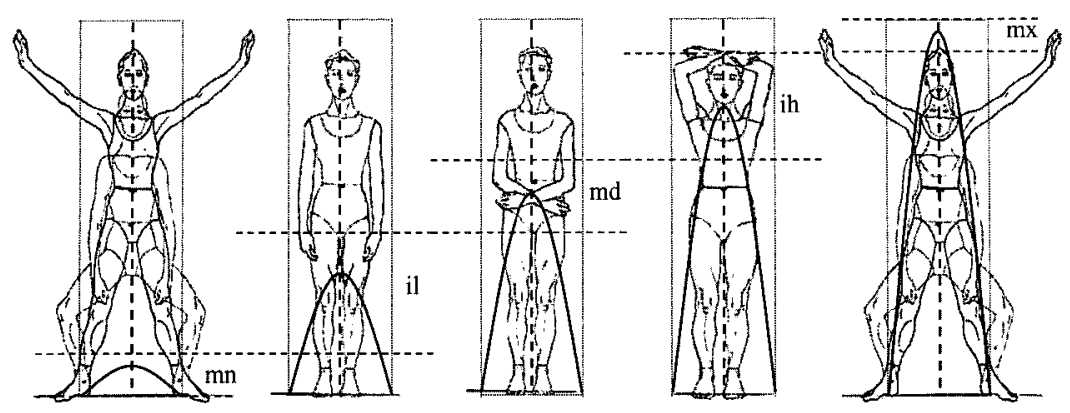

Figure 4. Derivation of a Visual Element Representing Quality $q_{i}^{R-S}$ of the $R$-S Elasticity and the Ranges of its Values.

the design of the visual representation of the qualities of the $C-E$ elasticity and how the respective composition rules for the $C-E$ elasticity in Table III are applied.

Similar to Figure 3, Figure 4 presents the logic behind the design of the visual representation of a quality of the $R-S$ elasticity and how the respective composition rules for the $R$-S elasticity in Table III are applied. In order to enable a comparison between computer-and human-based assessments, computer generated values are converted into a partially ordered categorical values as follows:

$$
q_{i}=\left\{\begin{array}{c}
m n, \text { if the estimate of a quality } \in[0,0.1[ \\
i l, \text { if the estimate of a quality } \in[0.1,0.4[ \\
m d, \text { if the estimate of a quality } \in[0.4,0.6[ \\
i h, \text { if the estimate of a quality } \in[0.6,0.9[ \\
m x, \text { if the estimate of a quality } \in[0.9,1]
\end{array}\right.
$$

In expression $6 \mathrm{mn}$ denotes 'minimum', il - 'intermediate-low', $m d$ - 'middle', ih 'intermediate-high', and $m x$ denotes 'maximum'. The order and the semantics of these

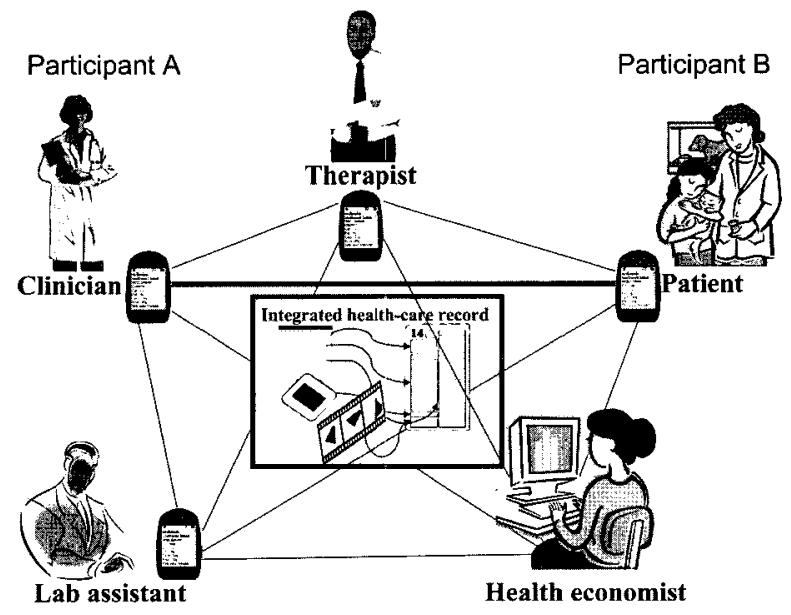

Figure 5. Similar to Collaborative Design, Modern Healthcare Domain Involves Interactions between Participants with Different Backgrounds. 
values for the qualities of respective elasticities are shown in Figure 3 and Figure 4. The source-target mapping in Figure 2 considers only three of these values -minimum, middle and maximum, which is sufficient for illustrating the idea without cluttering the picture.

As shown in Figure 2, the visual elements that represent the qualities of the $C-E$ and $R$-S elasticities are combined in a production element - the visualisation unit which represents an interaction segment. An interaction session is a collection of interaction segments; hence, it is represented by a collection of production elements that constitutes a production (see Figure 2). Productions are visual expressions. They provide a rich and compact view of the different sets of interactions, allowing to

- grasp the macro-picture of the interaction flow;

- perform comparative analysis of different parts of the same interaction;

- perform comparative analysis of different interactions.

In the next section we present an example that demonstrates how productions presented on mobile devices can be utilised in practice.

\section{DEPTOYING INTERACTION VTSUALISATION ON MOBILE DEVICES IN TFE I-WIEALTH DONARN}

The importance of practitioner-patient interactions has been recognised with the development of relationship-centred healthcare (see [Wylie and Wagenfeld-Heintz 2004] for a comprehensive literature review of the area). Similar to collaborative design [Maher et al. 2000] such interactions may involve parties with different backgrounds, as illustrated in Figure 5 . Further in this section we focus only on the practitioner-patient interaction (the thick line in Figure 5). Following the labels Participant A and Participant B in Figure 5, further in the section, where needed, practitioner and patient actions are labeled with "A" and "B", respectively.

In order to demonstrate a range of capabilities on mobile devices we bring elements from the analysis of four cases in health care, rather than focusing on the comprehensive analysis of a single case. The health care scenarios and interacting

Table IV. Health Care Scenarios Considered in this Section.

\begin{tabular}{|c|l|}
\hline Cases & \multicolumn{1}{|c|}{ Description } \\
\hline I & $\begin{array}{l}\text { Patient self management: Aging female patient assessed for discharge from hospital. } \\
\text { Interacting parties: Occupational therapist and Patient. }\end{array}$ \\
\hline II & $\begin{array}{l}\text { Culturally sensitive and marginalised healthcare: Developmental screening of a child to } \\
\text { assess cognitive abilities, specifically spatial reasoning and spatial memory. } \\
\text { Interacting parties: Occupational therapist and Patient. }\end{array}$ \\
\hline III & $\begin{array}{l}\text { Patient-healthcare professional communication: A clinician-patient dialogue to inform } \\
\text { the patient of decisions regarding choices in the patients' health journey. } \\
\text { Interacting parties: Clinician and Patient. }\end{array}$ \\
\hline IV & $\begin{array}{l}\text { Informed Participative Decision Making: A doctor-patient follow up post-operative } \\
\text { consultation. } \\
\text { Interacting parties: Medical specialist and Patient. }\end{array}$ \\
\hline
\end{tabular}


Table V. A fragment of the Action Sequences in Segment 1 of Practitioner-patient (A-B) Interaction in Case I.

\begin{tabular}{|l|l|l|}
\hline \multicolumn{1}{|c|}{ Action sequences } & \multicolumn{1}{c|}{ Subtasks } & \multicolumn{1}{c|}{ Decision forks } \\
\hline A1-A4:B1 & Introduction to patient & A4-DF Root \\
\hline A5-A8:B2-B3:A6-A7:B4:A6-A7:B4:A8:B5-B6 & Establish motivation & A5-DF1 \\
\hline A9:B7-B8:A10-A11:B9-B10:A12:B11B12:A13:B13 & Ability to self-manage & A9-DF2 \\
\hline
\end{tabular}

participants are briefly described in Table IV. In each case we have two participants interacting at one time. The current version of presented techniques can operate with more than two participants in the process, e.g., a few different healthcare professionals and a patient, or a healthcare professional, a patient and parent, with the limitation that only two parties are interacting at any one time.

The data source of each case is a video stream. These data are segmented into action sequences. Each segment is defined by a root decision fork - a point in the interaction sequence that marks the action that initiates the task or goal of a segment. Table V shows a sample of segmented data - the first segment of Case I. The parameters of these sequences are used in equations 1-5 to compute the values of the qualities in a production element for each segment.

Figure 6 and Figure 7 demonstrate diverse information views of the interaction profiles of patient-practitioner sessions, presented on mobile devices at the point of decision making. Figure 6 presents interaction profile views in a single session - in this example Case I, which deals with the suitability of an elderly patient for discharge from hospital to her own home where she lives alone. The interaction follows the assessment of the patient by obtaining some measure(s) of "fitness". The patient and practitioner interact through actions that are verbal and non-verbal. Figure $6 \mathrm{a}$ presents the production element corresponding to the interaction segment in Table V. The inclusion of the video is optional, subject to legal agreements. In this view the display area under the production element operates in several modes,

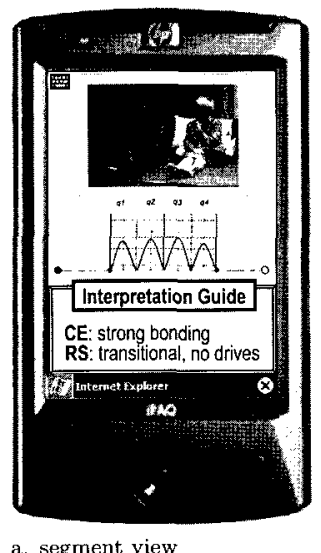

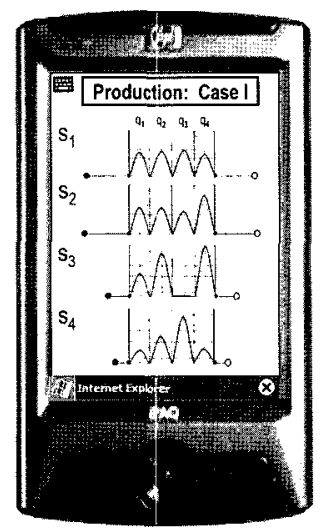

b. production view

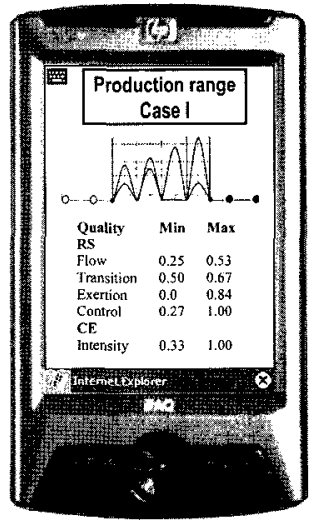

c. range view

Figure 6. Examples of Singe Case Views of the Information about Interactions that is Available to the Parties. 


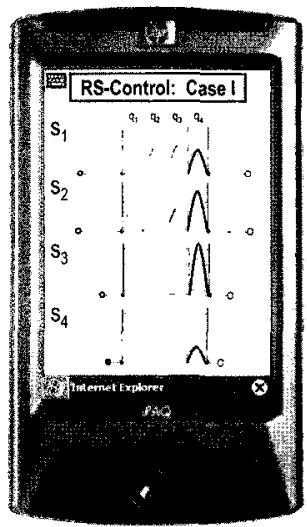

a. $q_{4}$-slice comparison

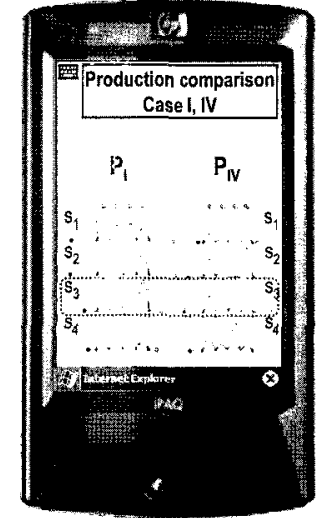

b. production comparison

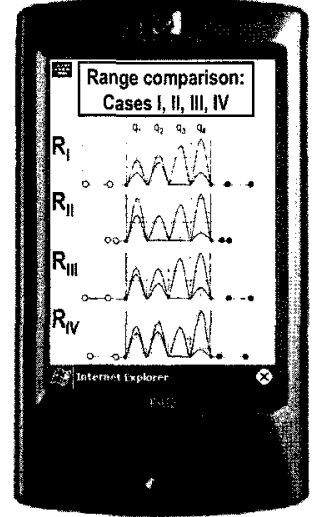

c. range comparison

Figure 7. Examples of Different Comparison Modes.

presenting: (i) raw segment data, similar to the action sequences in Table V; (ii) the values of the qualities and some statistics or; (iii) brief indicative interpretation about the interaction in the segment in terms of the elasticities, based on the values of the qualities (the example shown in Figure 6a). The production view in Figure 6b shows the entire interaction in Case I, which includes four segments, labelled as $S_{1}$ through $S_{4}$. In this production, flow $\left(q_{1}\right)$ indicates an average communication between the parties; transition $\left(q_{2}\right)$ indicates that communication between parties occurred with reasonably quick responses to actions of fairly short lengths; exertion $\left(q_{3}\right)$ indicates the complex level of interaction as it shows greater effort, especially in the last segment; control $\left(q_{4}\right)$ shows some flexibility in the middle of the session (segments 2 and 3 ) with a dominance of the practitioner towards the end. Figure $6 \mathrm{c}$ shows a visual summary of the interaction profile in the production range element, which indicates the ranges for each of the qualities during the interaction session. The options in this view for the display area under the production range element are similar to the modes of the above discussed production element view. In Figure $6 \mathrm{c}$ it shows the range statistics mode.

In addition to the interactions within a single session, practitioners and patients may be interested in comparison of the interactions in different sessions, for example, in several sessions during the treatment of the same clinical condition, or the interactions of the patient with different practitioners during the treatment. Alternatively, the comparison of the interaction profiles in different scenarios within the same domain can be of interest for the training of practitioners. In our example, the cases described in Table $\mathrm{V}$ can be viewed as an educational set for a practitioner in the occupational therapy. In line with this, Figure 7 demonstrates a sample of views that present different perspectives for comparison of several interaction profiles in a single display.

Figure 7a shows an example of comparison along a single quality within a session; in this example it is the control quality in Case I. These types of views offer comparison of one or more qualities, switching the rest of them in background (context) mode or 
taking them completely off the display. This is on example of support for visual analytics techniques that emphasise the dynamics along one or more dimensions and using the information about the other dimensions as the "context". Figure $7 \mathrm{~b}$ shows an example of comparison of productions representing the interactions of two different cases - Cases I and IV. The session in Case IV encapsulates the interaction between a doctor and a patient during a post-operative consultation. In this session the practitioner is dominant in the interaction process, discredits the patient's account of her post-operative experiences, and as a consequence reaches the wrong diagnosis. This session is an example of a poor communication. The comparative display mode offers comparison of interaction profiles in terms of complete productions and pairs of segments. Though the segmentation of different sessions depends on the structure of the session, this view provides a qualitative high level comparison. In Figure $7 \mathrm{~b}$, the comparison of interaction segments $P_{I}-S_{3}$ and $P_{I} V-S_{3}$ shows that in $P_{I}-S_{3}$ the qualities of effort shape perform with irregular profile with exertion at min and control at max, indicating a clearly defined direction of the interaction. In $P_{I} V-S_{3}$ the qualities are clustered much closer together with all close to average, indicating less flexibility in the interaction process. The two segments selected for comparison, can be displayed in separate display views where the display area under the production elements can display a comparison between the values or indicative interpretation of the respective productions for comparison. Comparisons across cases can include comparison between range elements as shown in Figure $7 \mathrm{c}$.

\section{CONCLUSIONS}

Interactions play central role in our technologically enriched and enabled life. We interact with our colleagues and professional team members in the office, with our doctors during the visits to healthcare practices, with our lecturers and classmates in university and in the virtual environments that have become an integrated part of contemporary learning experience. If the spaces where the interactions happen are equipped with a recording equipment, or the interactions are facilitated by information technology, we can have access to rich data about them and we can incorporate either these data in the respective organisational or personal data sets or the information extracted from the data. In this paper we presented a technology for visualisation on mobile devices of information about the dynamics of unfolding of interactions, extracted from such data sets. This technology includes

- a methodology for expressing interactions dynamics with constructs derived from the physicality of human movement;

- computational models of these constructs;

- graphical models of these constructs, where the visual shape and behaviour of their elements are aligned to certain aspects of human movements, as these aspects are encoded in our common knowledge [Pheifer and Bongard 2007];

- a set of display views for mobile devices that enable different tasks in utilisation of the information about interactions at the point of decision making;

- interpretation guidelines for the resultant patterns.

The paper formulated the requirements for such visualisations on mobile devices 
and demonstrated a technology that addresses compliance with these requirements. Proposed visual elements, their aggregation and other supporting operations present the complex dynamics of interactions in compact and condensed visual form on mobile devices. This technology enables several analytics tasks, including:

- exploration;

- comparison;

- summarisation;

- quality-specific analysis;

- tracking of particular patterns; and

- many more operations over the interaction profiles.

The major limitation in the current implementation is the data segmentation process, which is a semi-automatic procedure, where the human intervention depends on the scenario where the data is collected and the type of data collected. For example, the segmentation of video data usually requires more human involvement than the segmentation of text-based data collected in chat rooms and synchronised with other related documents. This limitation introduces a substantial lag in the delivery of information, which is important if such delivery is required in real-time.

Nevertheless, the capability of the proposed technology to document the dynamics of interactions between involved parties has the potential to extend the information that goes into the electronic records in various fields involving interactions; for example, in electronic health records in contemporary health care, or the materials that support various online and mobile learning strategies and assessment in contemporary education.

\section{ACRNOWLEIDGENENTS}

This research is supported by the University of Technology, Sydney and the University of Western Sydney.

\section{TRERERENCES}

BILLET, S. 2008. Learning through work: exploring instances of relational interdependencies. International Journal of Educational Research 47, 232-240.

BochNer, A. P., E. P. KAMINSKI, AND M. A. FITZPATRICK. 1977. The conceptual domain of interpersonal communication behavior; a factor-analytic study. Human Communication Research 3, 4, 291-302.

BradneR, E., W. A. KellogG, AND T. ERICKson. 1999. The adoption and use of babble: A field study of chat in the workplace. In Proceedings of the Sixth European Conference on Computer Supported Cooperative Work, ECSCW 99. Springer Netherlands: Copenhagen, Denmark, $139-158$.

CHANDLER, D. 2004. Semiotics: The Basics. Routlege.

CHEN, C. 2004. Information Visualization: Beyond the Horizon. Springer, London.

CHERNOFF, H. 1973. The use of faces to represent points in $\mathrm{k}$-dimensional space graphically. Journal of the American Statistical Association 68, 342, 361-368.

DERAY, K. AND S. J. SMOFF, 2007. Designing a visual language for interaction representation based on aspects of human movement. In Visual Languages for Interactive Computing, F. Ferri, Ed. IGI Global, Hershey, PA, 205-231.

DERAY, K. AND S. J. SimOFF. 2008. Visualising the dynamics of unfolding interactions on mobile 
devices. In Advances in Conceptual Modeling -Challenges and Opportunities, Proceedings of the ER 2008 International Workshop on Modeling Mobile Applications and Services (M2AS'08). Springer, Heidelberg, 238-247.

Dicke, C., S. Deo, M. BLlinghurst, N. ADAMs, AND J. LeHIKoINEN. 2008. Experiments in mobile spatial audio-conferencing: key-based and gesture-based interaction. In Proceedings of the 10th Conference on Human-Computer Interaction with Mobile Devices and Services Mobile HCI 2008. 2008, Amsterdam, the Netherlands. ACM.

FERREIRA DE OLIVEIRA, M. C. AND H. LeVKowTtZ. 2003. From visual data exploration to visual data mining: A survey. IEEE Transactions On Visualization And Computer Graphics 9, 3, 378-394.

GABB, R. AND S. KEATING. 2005. Work-based learning curricula in nursing: A literature review. Tech. rep., Victoria University: Melbourne, http://eprints.vu.edu.au/93/.http://eprints.vu.edu.au/ $93 \%$

GOLDER, S. A., D. WILKINSON, AND B. A. HUBERMAN. 2007. Rhythms of social interaction; messaging within a massive online network. In Proceedings of the 3rd International Conference on Communities and Technologies (CT2007). East Lansing, MI.

GREENFIELD, A. 2006. Everyware: The Dawning Age of Ubiquitous Computing. New Riders, Berkeley, CA.

INSELBERG, A. 1997. Multidimensional detective. In Proceedings of the IEEE Symposium on Information Visualization. Inselberg, A. Multidimensional detective, in Proceedings of the IEEE Symposium on Information Visualization. 1997: IEEE Press.

LAKoFf, G. AND M. JoHnson. 1980. Metaphors We Live By. University of Chicago Press, Chicago.

LoUwerse, M. M., A. C. Graesser, S. LU, AND H. H. MitChELL. 2005. Social cues in animated conversational agents. Applied Cognitive Psychology 19,693-704.

Maher, M. L., S. J. SimofF, AND A. Cicognani. 2000. Understanding virtual design studios. Springer-Verlag, London, UK.

Newlove, J. AND J. Dalby. 2004. Laban for All. Nick Hern Publishers, London.

PheIFER, R. AND J. BonGARD. 2007. How the Body Shapes the Way We Think. MIT Press, Cambridge, MA.

POUSMAN, Z. AND J. STASKO. 2006. A taxonomy of ambient information systems: four patterns of design. In Proceedings of the Working Conference on Advanced Visual Interfaces. ACM, Venezia, Italy.

PRETORIUS, A. J. 2005. Visual analysis for ontology engineering. Journal of Visual Languages and Computing 16, 359-381.

SIMOFF, S. AND F. SUDWEEKS. 2007. The language of leaders: Identifying emergent leaders in global virtual teams. In Linguistic and Cultural Online Communication Issues in the Global Age, K. St Amant, Ed. Idea Group, 93-111.

SimoFF, S. J. 2001. Towards the development of environments for designing visualisation support for visual data mining. In Proceedings of the International Workshop on Visual Data Mining VDM@PKDD2001. Freiburg, Germany.

SIMOFF, S. J. 2008. Form-semantics-function -a framework for designing visual data representations for visual data mining. In Visual Data Mining: Theory, Techniques and Tools for Visual Analytics, S. J. Simo., M. H. Böhlen, and A. Mazeika, Eds. Springer Verlag, Heidelberg, 3045.

STMOFF, S. J. AND M. L. MAHER. 2000. Analysing participation in collaborative design environments. Design studies 21, 119-144.

TANNEN, D. 2005. Conversational Style: Analyzing Talk among Friends. Oxford University Press.

THomas, J. J. AND K. A. COOK. 2005. Illuminating the Path: The Research and Development Agenda for Visual Analytics. IEEE CS Press.

WYLIE, J. L. AND E. WAGENFELD-HEINTZ. 2004. Development of relationship-centered care. Journal for Healthcare Quality 26, 1, 14-21. 

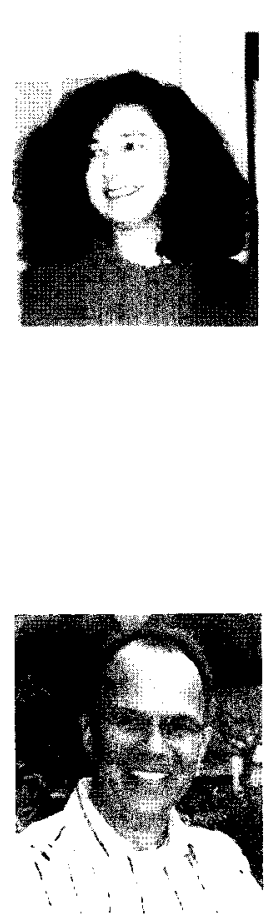

Kristine Deray is a Senior Lecturer in Design at the University of Technology, Sydney. She has an interdisciplinary background of design computing, spatial and performance design, dance and movement studies. She is particularly engaged with the exploration and the application of ambient visualization systems, based on physical metaphors, in the areas of mobile computing systems and 3D immersive virtual worlds as well as the capturing and utilization of information in relation to the dynamics of interactions. She is a core member of the Locative Design Lab (LDL) Research Group, and the Creative and Civil Societies at UTS. She has published in significant conferences both nationally and internationally focusing upon human usability technologies for consumer/patient empowerment in health care and pervasive service industries. Kristine is a chief investigator on a governmental community health project with Health Services Network, (HsNET) focused on the social construction of knowledge and community participation in health services, derived from digital stories.

Simeon Simoff is a Professor of Information Technology and Head of the School of Computing and Mathematics, University of Western Sydney. $\mathrm{He}$ is also an adjunct professor at the University of Technology, Sydney (UTS). Prior to this, he was a professor of information technology at the Faculty of Information Technology, UTS, where he established the eMarkets research program, currently running between UWS, UTS and UNSW (e-markets.org.au). $\mathrm{He}$ is also co-director of the Institute of Analytics Professionals of Australia and Series Editor of the ACS Publication Series "Conferences in Research and Practice in Information Technology". From 2000 to 2005 he was the associate editor (Australia) of the ASCE International Journal of Computing in Civil Engineering. He is founder and chair of the ACM SIGKDD Multimedia Data Mining workshop series MDM@KDD, the Australasian Data Mining Conference series AusDM, and the Visual Data Mining international workshop series at ECMLPKDD and ICDM events. His research interests include data analytics and data mining, human-computer systems, design computing and virtual worlds. 